POS PROCEEDINGS

\title{
Theory Predictions for LHC Higgs Boson Production
}

\author{
Franz Herzog ${ }^{* \dagger}$ \\ CERN \\ E-mail: franz.herzog@cern.ch
}

I discuss the current theoretical uncertainty on the inclusive Higgs boson cross section and review recent progress towards improving it by including N3LO corrections.

The European Physical Society Conference on High Energy Physics

18-24 July, 2013

Stockholm, Sweden

*Speaker.

†Preprint number: CERN-PH-TH/2013-273 


\section{Introduction}

With the $13 \mathrm{TeV}$ LHC we are about to enter a new era of Higgs Precision Physics. It is therefore a good time to revise our best theoretical predictions for the Higgs boson cross section and reassess our uncertainties. In this talk I focus on the gluon fusion channel, not only because it is the dominant Higgs boson production channel in the Standard Model, but also because the perturbative convergence of this channel is particularly slow. Due to its importance we now know a large variety of significant corrections to this observable. In fixed order perturbation theory we know it at next-to-next-to-leading order (NNLO) in the infinite top quark mass approximation of QCD $[1,2,3,4,5,6]$. The exact top mass dependence is known at next-to-leading order (NLO) [7, 8] and power corrections are known at NNLO [9, 10, 11, 12, 13]. Further more electro-weak(EW) corrections and mixed QCD-EW corrections have also been computed [14, 15, 16, 17, 18, 19, 20]. Also beyond fixed order much is known like the re-summation of soft gluons [21, 22] and a resummation of $\pi^{2}$ s [23].

\section{How accurately do we know the inclusive Higgs boson cross section?}

At $8 \mathrm{TeV}$ the inclusive Higgs cross section was independently estimated by two groups [25, 24]. To get a feeling where possible theoretical subtleties may lie we shall in the following compare the two predictions and identify the origin of possible discrepancies. Both predictions include all relevant electro-weak and QCD effects at fixed order. But [25] uses a lower central renormalization scale at $\mu_{R}=m_{H} / 2$ and includes only fixed order corrections, while [24] uses $\mu_{R}=m_{H}$ but includes also threshold resummation. Both references agree that the parton distribution function (PDF) uncertainties are between $7-8 \%$, but have somewhat different predictions for the scale uncertainties. In [24] these are estimated to lie around 7\%. In contrast [25] find a scale uncertainty around $9 \%$. Nevertheless the central predictions of both agree within their quoted uncertainties.

So it appears that the scale uncertainties are slightly reduced by the inclusion of threshold resummation. It has been argued that the scale uncertainties can be reduced even further by taking into account the resummation of $\pi^{2} \mathrm{~s}$ [23]. On the contrary [25] argues that the perturbative series converges faster for scale choices around $\mu_{R}=m_{H} / 2$, as can be seen from Figure 1. Assuming that this pattern continues also at yet higher orders it should therefore lead to smaller perturbative corrections. Indeed it is interesting to note that for scale choices around $\mu_{R}=m_{H} / 2$ the effects of soft gluon resummation or the resummation of $\pi^{2} \mathrm{~s}$ are numerically reduced as can be seen from scale variations in $[21,23]$.

The PDF uncertainties are likely to be reduced further with LHC jet data, however the only reliable way to reduce scale uncertainties is by including further higher order contributions. So far only an approximation of the next-to-next-to-next-to-leading order (N3LO) correction is known [26], which has been obtained by first truncating and then matching BFKL and threshold resummations. There are subtleties involved in this procedure but the authors argue that by exploiting the analyticity of the Mellin space cross section these can be overcome. In Figure 1 we show the scale variation of the Higgs cross section for initial state gluons only[30]. The plot contains fixed order results for LO, NLO, NNLO and the N3LO approximation. The pink lines represent different possibilities for N3LO scale variations given that the scale dependent logarithmic terms are fixed 


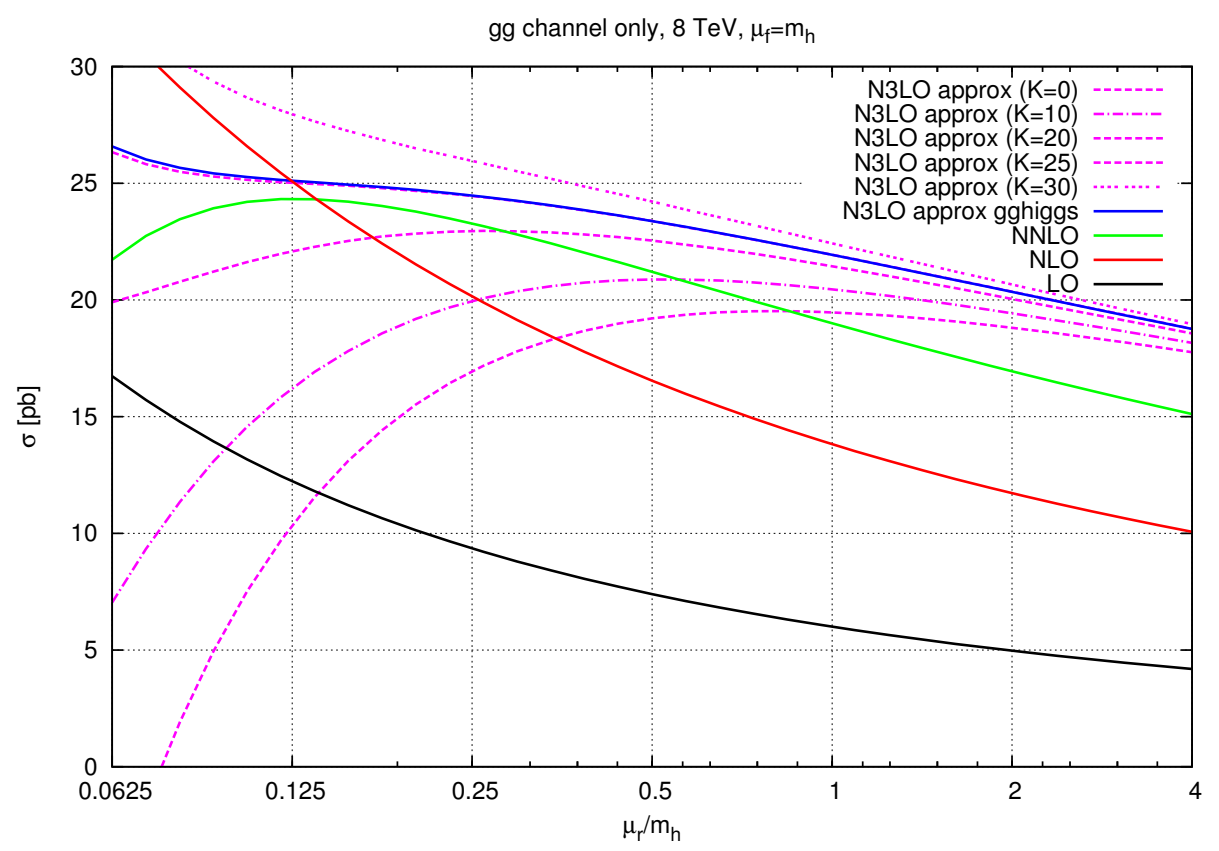

Figure 1: Renormalization Scale variations taken from [30]. The pink lines correspond to different possible scale variations at N3LO. The blue line correspond to the approximation of [26]

completely by renormalization group invariance but assuming otherwise arbitrary non-scale dependent terms. Maybe we should emphasise here that if the Higgs boson cross section is reasonably perturbative then we may expect it to be considerably flatter than the NNLO curve and therefore lie somewhere in the range between $K \sim 15$ and $K \sim 25$. The upper bound of which is where it is set by the approximate N3LO prediction of [26]. If one would adopt a central scale of $\mu_{R}=m_{H}$ for the NNLO, then this would indeed lead to an increase of about $17 \%$. The situation is significantly improved for $\mu_{R}=m_{H} / 2$ but still leads to about $10 \%$ enhancement.

Figure 1 also allows one to read off the scale uncertainty that one can expect at N3LO, which lies around $4-6 \%$ according to a more or less perturbative N3LO scenario.

\section{Status of N3LO}

In the following we briefly summarise the current progress towards computing the N3LO correction exactly. This correction is naturally split into 5 different contributions, classified by the number of cut propagators present. Sample Feynman diagrams contributing to each of these are illustrated in Figure 2. Even though one may assume that all master integrals should be solvable with the method of differential equations, their sheer number and the possible entanglement of the linear systems to be encountered may still make an evaluation extremely difficult. Alternatively it is well known, from experience at lower orders, that a series expansion around the threshold converges quickly. Thus a very good approximation to the full N3LO correction, can be obtained already by including only a few terms in the threshold expansion. For some of the contributions 

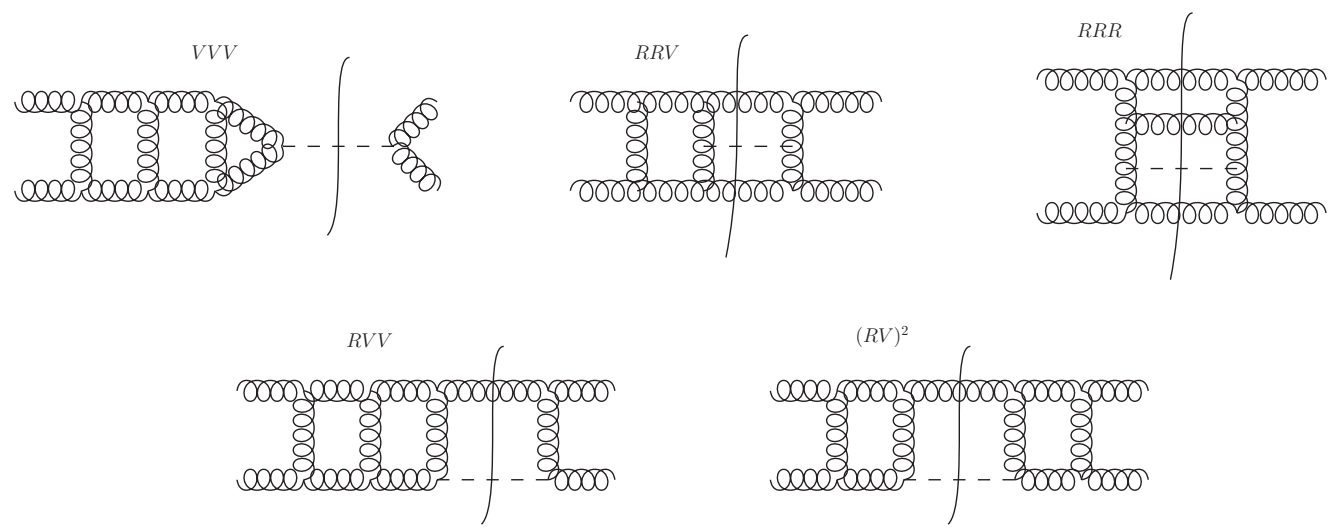

Figure 2: Sample Diagrams of different contributions at N3LO

this may well turn out to be the method of choice if a complete analytic evaluation turns out to be too challenging. Let us now summarize the current state of the art.

- Collinear and UV counter terms:

These are known in the literature [27, 28, 29, 30].

- Triple Virtual (VVV):

The three loop contribution is already known in the literature [31, 32].

- Real Double Virtual (RVV):

This contribution is not known at this point, nevertheless many of the ingredients needed are already available, this includes the two-loop amplitudes [33] as well as the soft current at two loop [34], which also constitutes the leading term in a threshold expansion.

- Real Virtual squared (RV) ${ }^{2}$ :

This contribution is now known [35].

- Double Real Virtual (RRV):

This contribution is not known yet but work is ongoing.

- Triple Real (RRR):

For this contribution the first two terms in an expansion around threshold are known [36]. The methods developed in this reference can also be used to compute further terms in the threshold expansion.

\section{Conclusions}

We have summarized the current status of the art of high precision predictions for inclusive Higgs production in the Standard Model. In order to match the expected accuracy of the coming LHC $13 \mathrm{TeV}$ data the Higgs boson coupling extractions it will be necessary to further reduce theoretical uncertainties. Progress towards this goal is well underway and one can anticipate that the N3LO correction will be completed in the next few years thereby also shedding new light on the validity of current predictions and their associated uncertainties. 


\section{References}

[1] S. Dawson, Nucl. Phys. B 359 (1991) 283.

[2] A. Djouadi, M. Spira and P. M. Zerwas, Phys. Lett. B 264 (1991) 440.

[3] D. Graudenz, M. Spira and P. M. Zerwas, Phys. Rev. Lett. 70 (1993) 1372.

[4] R. V. Harlander and W. B. Kilgore, Phys. Rev. Lett. 88 (2002) 201801 [hep-ph/0201206].

[5] C. Anastasiou and K. Melnikov, Nucl. Phys. B 646 (2002) 220 [hep-ph/0207004].

[6] V. Ravindran, J. Smith and W. L. van Neerven, Nucl. Phys. B 665 (2003) 325 [hep-ph/0302135].

[7] M. Spira, A. Djouadi, D. Graudenz and P. M. Zerwas, Nucl. Phys. B 453 (1995) 17 [hep-ph/9504378].

[8] U. Aglietti, R. Bonciani, G. Degrassi and A. Vicini, JHEP 0701 (2007) 021 [hep-ph/0611266].

[9] R. V. Harlander and K. J. Ozeren, Phys. Lett. B 679 (2009) 467 [arXiv:0907.2997 [hep-ph]].

[10] R. V. Harlander and K. J. Ozeren, JHEP 0911 (2009) 088 [arXiv:0909.3420 [hep-ph]].

[11] R. V. Harlander, H. Mantler, S. Marzani and K. J. Ozeren, Eur. Phys. J. C 66 (2010) 359 [arXiv:0912.2104 [hep-ph]].

[12] A. Pak, M. Rogal and M. Steinhauser, Phys. Lett. B 679 (2009) 473 [arXiv:0907.2998 [hep-ph]].

[13] A. Pak, M. Rogal and M. Steinhauser, JHEP 1002 (2010) 025 [arXiv:0911.4662 [hep-ph]].

[14] A. Djouadi and P. Gambino, Phys. Rev. Lett. 73 (1994) 2528 [hep-ph/9406432].

[15] U. Aglietti, R. Bonciani, G. Degrassi and A. Vicini, Phys. Lett. B 595 (2004) 432 [hep-ph/0404071].

[16] G. Degrassi and F. Maltoni, Phys. Lett. B 600 (2004) 255 [hep-ph/0407249].

[17] S. Actis, G. Passarino, C. Sturm and S. Uccirati, Nucl. Phys. B 811 (2009) 182 [arXiv:0809.3667 [hep-ph]].

[18] S. Actis, G. Passarino, C. Sturm and S. Uccirati, Phys. Lett. B 670 (2008) 12 [arXiv:0809.1301 [hep-ph]].

[19] C. Anastasiou, R. Boughezal and F. Petriello, JHEP 0904 (2009) 003 [arXiv:0811.3458 [hep-ph]].

[20] W. -Y. Keung and F. J. Petriello, Phys. Rev. D 80 (2009) 013007 [arXiv:0905.2775 [hep-ph]].

[21] S. Catani, D. de Florian, M. Grazzini and P. Nason, JHEP 0307 (2003) 028 [hep-ph/0306211].

[22] S. Moch and A. Vogt, Phys. Lett. B 631 (2005) 48 [hep-ph/0508265].

[23] V. Ahrens, T. Becher, M. Neubert and L. L. Yang, Eur. Phys. J. C 62 (2009) 333 [arXiv:0809.4283 [hep-ph]].

[24] D. de Florian and M. Grazzini, Phys. Lett. B 718 (2012) 117 [arXiv:1206.4133 [hep-ph]].

[25] C. Anastasiou, S. Buehler, F. Herzog and A. Lazopoulos, JHEP 1204 (2012) 004 [arXiv:1202.3638 [hep-ph]].

[26] R. D. Ball, M. Bonvini, S. Forte, S. Marzani and G. Ridolfi, Nucl. Phys. B 874 (2013) 746 [arXiv:1303.3590 [hep-ph]].

[27, 28, 29, 30]

[27] A. Pak, M. Rogal and M. Steinhauser, JHEP 1109 (2011) 088 [arXiv:1107.3391 [hep-ph]]. 
[28] C. Anastasiou, S. Buehler, C. Duhr and F. Herzog, JHEP 1211 (2012) 062 [arXiv:1208.3130 [hep-ph]].

[29] M. HÃúschele, J. Hoff, A. Pak, M. Steinhauser and T. Ueda, Phys. Lett. B 721 (2013) 244 [arXiv:1211.6559 [hep-ph]].

[30] S. Buehler and A. Lazopoulos, JHEP 1310 (2013) 096 [arXiv:1306.2223 [hep-ph]].

[31] P. A. Baikov, K. G. Chetyrkin, A. V. Smirnov, V. A. Smirnov and M. Steinhauser, Phys. Rev. Lett. 102 (2009) 212002 [arXiv:0902.3519 [hep-ph]].

[32] T. Gehrmann, E. W. N. Glover, T. Huber, N. Ikizlerli and C. Studerus, JHEP 1011 (2010) 102 [arXiv:1010.4478 [hep-ph]].

[33] T. Gehrmann, M. Jaquier, E. W. N. Glover and A. Koukoutsakis, JHEP 1202 (2012) 056 [arXiv:1112.3554 [hep-ph]].

[34] C. Duhr and T. Gehrmann, arXiv:1309.4393 [hep-ph].

[35] C. Anastasiou, C. Duhr, F. Dulat, F. Herzog and B. Mistlberger, arXiv:1311.1425 [hep-ph].

[36] C. Anastasiou, C. Duhr, F. Dulat and B. Mistlberger, JHEP 1307 (2013) 003 [arXiv:1302.4379 [hep-ph]]. 